\title{
http://dx.doi.org/10.35381/racii.v5i8.588
}

\section{Violencia económica y patrimonial. Acceso a una vida libre de violencia a las mujeres}

\section{Economic and patrimonial violence. Access to a life free of violence to women}

\author{
Viviana Leonor Maldonado-García \\ viviana.maldonado@ucacue.edu.ec \\ Universidad Cátolica de Cuenca, Cuenca \\ Ecuador \\ https://orcid.org/0000-0003-1841-2911 \\ Juan Carlos Erazo-Álvarez \\ icerazo@ucacue.edu.ec \\ Universidad Católica de Cuenca, Cuenca \\ Ecuador \\ https://orcid.org/0000-0001-6480-2270 \\ Enrique Eugenio Pozo-Cabrera \\ epozo@ucacue.edu.ec \\ Universidad Católica de Cuenca, Cuenca \\ Ecuador \\ https://orcid.org/0000-0003-4980-6403 \\ Cecilia Ivonne Narváez-Zurita \\ inarvaez@ucacue.edu.ec \\ Universidad Católica de Cuenca, Cuenca \\ Ecuador \\ https://orcid.org/0000-0002-7437-9880
}

Recibido: 15 de noviembre de 2019

Aprobado: 17 de diciembre de 2019

\section{RESUMEN}

En el presente artículo de investigación se analiza como dentro de la legislación ecuatoriana existe una falta de protección a la violencia económica y patrimonial que sufren las mujeres, por lo cual se plantea una reforma al Código Orgánico Integral Penal que busque equipar los derechos humanos y de género en el Ecuador. La metodología investigativa partio desde lo cualitativo, utilizando métodos tales como análisis y síntesis 
bibliográficas, así como también histórico lógico e inductivo deductivo. En este sentido, se determina que con esta reforma planteada se asegurara la protección que las mujeres tendrán dentro de la legislación ecuatoriana será apegada a los principios y garantías constitucionales como acceso a la justicia, garantizado la tutela efectiva, lo que generara equidad en futuros casos.

Descriptores: Violencia económica patrimonial; Violencia contra las mujeres; Victimas; Derechos humanos; Constitución.

\begin{abstract}
In this research article, it is analyzed how within Ecuadorian legislation there is a lack of protection for economic and patrimonial violence suffered by women, which is why a reform of the Organic Integral Criminal Code that seeks to equip human and human rights is proposed. gender in Ecuador. The research methodology started from the qualitative, using methods such as analysis and bibliographic synthesis, as well as historical logic and deductive inductive. In this sense, it is determined that with this proposed reform, the protection that women will have within Ecuadorian legislation will be ensured will be attached to constitutional principles and guarantees as access to justice, guaranteed effective guardianship, which will generate equity in future cases. .
\end{abstract}

Descriptors: Economic and patrimonial violence; Violence against women, Victims; human rights; Constitution

\title{
INTRODUCCIÓN
}

En el año 2008, la reforma a la Carta Magna del Ecuador garantiza un Estado constitucional de derechos y justicia, estableciendo como uno de los grupos de atención prioritara las vícitmas de violencia intrafamililar, por parte del Estado en su entorno público y privado (Asamblea Nacional Constituyente, 2008). Uno de los derechos de todas las personas es la tutela judicial efectiva, en los procesos de agresiones realizadas a las víctimas de violencia se debe implementar vías legales eficaces a la justicia que no menoscaben o anulen el ejercicio de ese derecho a la integridad de la mujer como ser humano, evitar la revictimización y afianzar el respeto a la normativa internacional en la no discriminación.

Las estrategias y acciones unificadas por los movimientos de mujeres y alianzas con organizaciones políticas representadas por la Asamblea promovieron que se les incluya 
en varios derechos a las mujeres. Es por eso que, en varias décadas las mujeres has sufrido varios tipos de violencia, entre ellos los más comunes son: violencia física, psicológica y sexual, dejando que sus derechos sean vulnerados, por considerarlas inferiores a los hombres por el sistema machista y patriarcal de nuestra sociedad (Aldana Zavala \& Valles González, 2018).

En efecto, con la implementación de otros tipos de violencia que sufren las mujeres, se incorpora al ordenamiento jurídico el nuevo tipo de violencia económica y patrimonial, con el objeto de que las mujeres accedan a reclamar sus bienes muebles e inmuebles, objetos personales que por derecho les pertenecen, ya sean estos adquiridos en matrimonio, unión de hecho, o en relación de convivencia (Asamblea Nacional del Ecuador, 2018). En este caso, es necesaria la construcción explícita que trate la violencia patrimonial de un modo adecuado y eficaz para sancionar y erradicar la violencia económica y patrimonial, acorde al procedimiento expedito que tipifica y sanciona el Código Orgánico Integral Penal (COIP) en los casos de violencia.

A pesar de todo lo logrado y generado por el Estado ecuatoriano, por las organizaciones de mujeres y la sociedad entre sí, actualmente la reforma del artículo 159 del COIP, aún se puede evidenciar ligeramente el tema de investigación, respecto al escenario vivido por la violencia estructural contra las mujeres de índole intrafamiliar , negando la potestad de denunciar dichas agresiones ante los órganos jurisdicionales correspondientes, para asegurar su bienestar y de sus familias.

Dicha reforma data con fecha 05 de febrero del año 2018, con la finalidad de abarcar en parte lo relativo a la violencia patrimonial en el artículo 159 inciso tercero del COIP, la misma que no satisface las necesidades de su entorno patrimonial y económico, es por eso que las mujeres son víctimas a diario de este tipo de violencia, por lo que debería existir un reforma integral que haga relación al procedimiento adecuado para su respetiva aplicación y sanción, más aún sí consideramos que se ha dejado como una expresión teórica y vacía que en la práctica difiere de su esencia y propósito. 
Es obligación del Estado generar procesos que eviten la revictimización de las víctimas de violencia al momento de acudir a la administacion de justica. Los abusos y violaciones a los derechos humanos contra las mujeres deber ser resueltos y apartados de cualquier prejuicio ya sea de diversidad sexual o caulquier tipo, por lo que no se cuenta con una normativa adecuada que viabilice y reglamente el procedimiento para una correcta aplicación de la norma sustantiva respecto a la erradicación de la violencia económica y patrimonial, en este sentido, es importante establecer un mecanismo adecuado y eficaz, que avale el pleno cumplimiento de esta garantía constitucional y que su vulneración sea debidamente sancionada, es obligación del Estado garantizar el acceso a la justicia de quienes lo requieren ya sea en entidades públicas como en su vida cotidiana. Con estos antecedentes se plantea el problema de la presente investigación: ¿Cómo reformar las contravenciones de violencia contra la mujer o miembros del núcleo familiar incorporado textualmente la violencia Económica y Patrimonial en el COIP?

Para tal efecto el objetivo es proponer la garantización del acceso a la justicia y a una vida libre de violencia de las mujeres en el COIP.

\section{DESARROLLO}

\section{El rol de las mujeres al acceder a la justicia; una problemática social y cultural envuelta en el sistema patrialcal}

Las relaciones de poderes hacia las mujeres, es una problemática social y cultural, que implican desigualdad entre hombres y mujeres. Históricamente las mujeres han sido vulneradas en sus derechos y consideradas inferiores, debido a las construcciones sociales del sistema patriarcal ecuatoriano, en el cual las mujeres han sido víctimas de diferentes tipos de violencia. Las mujeres han sido excluidas en la sociedad y en sus derechos, esto es debido a que los imaginarios sociales han puesto a las mujeres al cuidado de su familia, marginándoles de toda relación social, política y económica dejando en subordinación a la mujer (Facio y Fries, 2005). Al respecto, Benhabib (1992) 
afirma: "El género opera como una estructura de poder, de igual forma que la clase, la raza o la cultura "(p.38). Al hacer referencia a una estructura de poder Sendón de León (2003) afirma que las sociedades han categorizado jerárquicamente al hombre y a la mujer, es así como las mujeres han sido subordinadas y dominadas por los hombres, tan solo por el hecho de ser mujer, sin tener decisión sobre sus derechos. Dentro de esta perspectiva, Butler (2018) manifiesta:

Cuando los científicos sociales hablan del género como un factor o una dimensión del análisis, también se refieren a personas encarnadas como una marca de diferencia biológica, lingüística o cultural. En estos casos, el género puede verse como cierto significado que adquiere un cuerpo ya sexualmente diferenciado, pero incluso en ese caso ese significado existe únicamente en relación con otro significado opuesto. (p.59).

Cabe considerar, por otra parte, los mitos creados por la humanidad, que han ejercido los grandes poderes para el patriarcado, y así daría poder económico a los hombres sobre las mujeres, por lo que es aprovechado para poder ejercer autoridad y adueñarse de lo femenino, la mujer no ha representado para el hombre y la sociedad la importancia en el curso de la costumbre, en la actualidad todavía se escucha jlos hombres son los mejores jefes! ¡los hombres son los mejores médicos! ¡las mujeres solo sirven para las tareas de limpieza! ¡una mujer no debe exigir más de lo que el hombre le dé! ¡las mujeres se merecen ser golpeadas porque se portan mal! ¡los hombrecitos no lloran! (Prieto, 2005).

En síntesis, a pesar de todo lo logrado y generado por el Estado ecuatoriano, por los movimientos de mujeres y la sociedad entre sí, fomentando las políticas públicas y sociales desarrollando normativas de protección para que las mujeres tengan acceso a la justicia y se sancione los diferentes tipos de violencia, en el año 1995, por primera vez en el Ecuador se incluye y conceptualiza la violencia intrafamiliar e incluyendo la violencia física, psicológica y sexual, siendo así que, el Estado asumió una rectoría y responsabilidad civil creando las primeras Comisarias de la Mujer y la Familia en el Ecuador (Congreso Nacional, 1995). No obstante, Almeras y Calderón (2012) manifiestan: 
Son pocas las investigaciones que buscan establecer cuál es la medida del déficit de acceso a la justicia para las mujeres que han visto vulnerado su derecho a una vida libre de violencia. El interrogante que se plantea es hasta qué punto las personas que se encuentran en esa situación han podido activar los mecanismos institucionales existentes ya sean administrativos o judiciales. (p.86)

Por último, la Constitución de la República del Ecuador (CRE), los Tratados Internacionales ratificados por el Ecuador y demás normas referentes en violencia de género, no garantizan la eliminación y erradicación de todo tipo de violencia contra las mujeres; a la igualdad formal y material y no discriminación, de tal manera que se no se está garantizando en su totalidad la premisa constitucional que es el acceso a la justicia de las mujeres y a una vida libre de violencia de manera oportuna y eficaz (Naciones Unidas, 2002).

\section{Un análisis comparativo de legislaciones en Latinoamérica en referencia a la violencia de género}

A lo largo de la legislación ecuatoriana, el acceso a la justicia y a una vida libre de violencia de las mujeres ha tenido una gran relevancia, en este caso es necesario sintetizar como la normativa ha ido reformándose para garantizar un mecanismo de protección a las mujeres, con el objeto de priorizar que el Estado ecuatoriano dé una respuesta rápida, oportuna y eficaz a este grupo de atención prioritaria.

Los derechos de las personas establecidos en la CRE, son irrevocables y perennes, tienen así la garantía de velar por sus interés, de obtener resoluciones y sentencia fundadas en razón de la exigencia en reclamar sus derechos y garantías, sin ningún costo, que deben ser atendidos y agilitados por los servidores judiciales (Asamblea Nacional Constituyente, 2008).

Así mismo con referencia a la tutela efectiva, este derecho de protección no solo puede ser garantizado por el Estado, también tienen gran responsabilidad los operadores de justicia, a que se proteja la vulneración de los derechos humanos y garantías establecidos por la Constitución de la República del Ecuador (CRE) en sus diferentes 
etapas procesales, quienes deben garantizar el acceso a la justicia cuando lo requieran de manera oportuna, inmediata y eficaz (Guzmán, 2010). Cabe considerar, por otra parte, que el Estado ecuatoriano tiene la obligación de fomentar políticas públicas y sociales para que los sujetos que hayan sido vulneradas en sus derechos y garantías constitucionales tengan la facultad de reclamar la reforma, cumplimento y el perfeccionamiento de estos (Grijalva, 2012).

De esta manera, en la legislación ecuatoriana se incorporó el inciso tercero en el art 159 sin mencionar expresamente violencia económica y patrimonial, como un tipo de violencia estructural e independiente, con el objeto de que las mujeres puedan acceder a la justicia y a una vida libre de violencia. Por lo que es importante indicar lo que la Asamblea Nacional del Ecuador (2018) señala:

La persona que realice actos de sustracción, destrucción, retención de objeto, instrumentos de trabajo, documentos personales o bienes de la sociedad de hecho o conyugal, en los casos en que no constituya un delito autónomo tipificado en este Código, será sancionada con trabajo comunitario de cuarenta a ochenta horas y la devolución de los bienes o el pago en valor monetario de los mismos, y medida de reparación integral. (art.159, inc. tercero)

A continuación, en el cuadro 1 se demuestra, en forma sucinta que la mayoría de los países de Latinoamérica tienen legislación, que, comparada entre sí en lo referente al acceso a la justicia y a una vida libre de violencia de las mujeres. 


\section{Cuadro 1}

Análisis cualitativo de las legislaciones en Latinoamérica sobre el acceso a la justicia en violencia intrafamiliar.

\section{País}

Argentina

Venezuela

Guatemala

Colombia

Perú

Chile

Cuba

\section{Normativa}

La Ley 26.485 de protección integral para prevenir la violencia contra las mujeres del año 2009 en su artículo 5 numeral 4, aprueba la violencia patrimonial como tipo de violencia estableciendo en su legislación.

Establece definiciones específicas de violencia de familia, además se contemplan nuevas formas de violencia entre ellas la violencia patrimonial y económica en su artículo 15, numeral 12 de la Reforma a la ley Orgánica sobre el Derecho de las Mujeres a una Vida Libre de Violencia del año 2007.

En el decreto 22-2008, de la Ley contra el femicidio y otras formas de violencia contra la mujer, protege los derechos de la mujer incorporando la violencia económica.

$\mathrm{Se}$ instaura normas sancionatorias y preventivas para erradicar la violencia contra las mujeres y se implementa el artículo № 2 Violencia Patrimonial, en la Ley 1257 de 2008.

En el año 2015, la Ley 30364, permite sancionar varios tipos de violencia y aplica la violencia económica o patrimonial en su artículo 8 inciso b, como violencia contra las mujeres.

Especifican penas más graves en los tipos de violencia física y psicológica, reformando el articulo 14 de la Ley $N^{\circ} 20.066$ que constituye la Ley de Violencia Intrafamiliar.

La Ley 87 de 1999, protege los derechos humanos, y otros tipos de violencia familiar, sin embargo, no tipifica los diferentes tipos de violencia en la Ley 87 de 1999. 
En base al cuadro plasmado, los países como Argentina, Venezuela, Guatemala, Colombia agregan la violencia económica y patrimonial como un nuevo tipo de violencia hacia las mujeres, protegiendo los derechos humanos y generado que las mujeres tengan acceso a la justicia. En consecuencia, el Ecuador a recopilado estas normativas y ha generado políticas públicas en su mayoría para proteger los derechos que han sido vulnerados por el sistema patriarcal. Con referencia a Cuba y Chile, todavía se evidencia un retroceso en el tema de los derechos humanos, sin existir un avance en sancionar las agresiones provocadas por el círculo familiar y en relaciones de pareja.

Para finalizar, cabe acotar que para el mes de febrero del año 2018 hubo una reforma importante respecto de la ampliación en la parte adjetiva de las garantías de protección de los derechos de las mujeres y miembros del grupo familiar respecto del ámbito patrimonial, por interés del populismo político no se ha sociabilizado de la manera adecuada el art. 159 inciso tercero del COIP; sin embargo este articulado no indica de manera explícita que se trata de violencia patrimonial, lo que dejaría una puerta abierta para que se interprete de manera no apropiada. Se plantea una propuesta que reforme la ampliación en la parte sustantiva del art 159 numeral 3 del COIP, estableciendo de manera tácita y expresa el tipo de violencia económica y patrimonial, para suprimir y proteger las relaciones de poder que se dan entre lo femenino y masculino.

\section{Violencia patrimonial y económica; una desigualdad silenciosa que sufren las mujeres}

Existen varias corrientes que defienden la violencia patrimonial y económica como un problema social y cultural, entre las principales se encuentran: la violencia patrimonial tiene un problema fundamental que es la desigualdad de derechos, que se ejerce en el entorno de las mujeres, es una forma de controlar el dinero, se manifiesta en la relación de pareja, no se valora el trabajo doméstico porque no genera dinero, afecta a la económica de la mujer, lesiona los derechos de sus hijos e hijas. Este tipo de violencia para la sociedad todavía sigue siendo invisible, silenciosa ya que los hombres son 
quienes siguen manejando la mayor parte de los ingresos de la sociedad conyugal y ejercen poder de los bienes muebles e inmuebles (Proveyer y Romero, 2017).

De igual manera Munster (2017) señala: "las mujeres, en cambio, han sido caracterizadas como dependientes y sin autonomía para sus decisiones económicas" (p.178). En este mismo contexto, Deere, Contreras, y Twyman (2014) refieren que en el Ecuador mayor parte de las mujeres a pesar de tener pleno conocimiento sobre sus derechos en relativo a su patrimonio y economía, todavía existen brechas fundamentales sobre el discernimiento de la normativa vigente, en cuanto a la repartición justa de sus bienes, de tal forma que pueden sufrir otros tipos de violencia por parte de sus parejas, al ser vulnerados sus derechos constitucionales. Por otra parte, expresa Díaz (2019) que el sistema androcentrismo instaurado en las sociedades ya no solo afecta a las mujeres en relación con el dominio de poder, también admite que afecta y engloba un conjunto de retrocesos económicos que afectan a la evolución de la economía familiar.

Por lo antes dicho, se puede evidenciar que existen varios criterios similares a una clara definición del tipo de violencia económica y patrimonial, sin embargo, es necesario acotar, qué este tipo de violencia puede ser generalizado, normalizado y confundido por un tipo de violencia psíquica, dejando en la subordinación e indefensión de acceder a la justicia a las mujeres al reclamar sus derechos (López, 2017). En relación con la problemática expuesta, el Sistema de Información Científica (2015) afirma:

Algunas mujeres comentaron que el miedo a sus exparejas les impidió concretar acuerdos económicos o fueron coaccionadas para aceptar acuerdos injustos, una de ellas comentó: pero sí, por el miedo que tengo, que tenía, bueno que tengo, fue que no llegamos a un acuerdo. (p.89)

A este respecto, los esfuerzos realizados por las organizaciones de mujeres, en la creación de políticas públicas en la eliminación y erradicación de todo tipo de violencia generada a la mujer, el Ecuador en el año de 1995, crea la normativa que fue más conocida como la Ley 103 implementando varios tipos de agresiones, las mismas que no satisficieron las necesidades de protección requeridas. 
La violencia física, es calificada como el contacto físico violento que sufren las mujeres, son los golpes, puntapiés, bofetadas, proporcionados por su pareja o miembros del núcleo familiar, incluso estas agresiones pueden ser causadas por armas blancas, lesionando así el cuerpo humano, causándoles hasta la muerte de quienes sufren esta violencia. La violencia psíquica, es la violencia más común que sufren las mujeres, debido a los estereotipos instaurados por el sistema machista patrialcal de la sociedad, en el cual desvaloriza a la mujer con epítetos descalificativos, agraviando así el estado emocional de la misma, y por último, la violencia sexual que sin duda alguna es la más alta violación al derecho humano, atenta al derecho sexual de la mujer a elegir con quién mantener relaciones sexuales en relación de pareja (Congreso Nacional, 1995). En síntesis y dentro de este marco, esta ley fue derogada en el año de 2014, y entro en vigor el COIP en fecha 10 de agosto del 2014, ampliando los conceptos de violencia en contra de las mujeres y a su vez convitiendolos en delitos y contravenciones, endureciendo las sanciones y las penas por cometer este tipo de agresiones, empero, en el mes de febrero del año 2018, el COIP fue reformado por tener deficiencias en estos articulados.

Ahora bien, en dicha reforma se implementa el inciso tercero en el art.159, en el cual no se indica de manera explícita, clara y precisa la terminología "violencia económica y patrimonial" como tipo de violencia, lo que dejaría sin acceso a la justicia a las mujeres y sin poder reclamar sus derechos constitucionales. A continuación, se muestra la figura 1 , donde consta la propuesta de proyecto de reforma a la Ley Organica Integral Penal. 


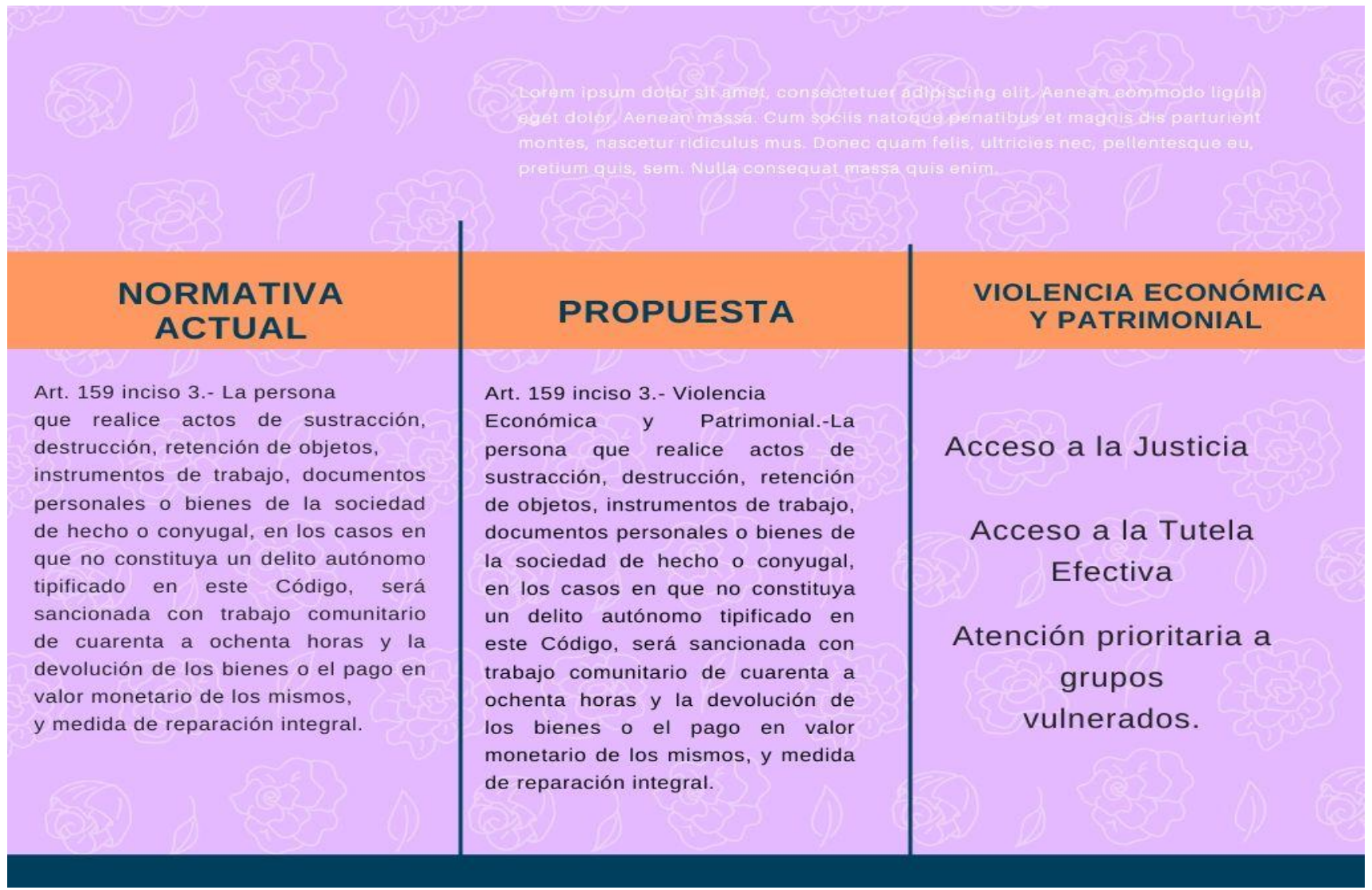

Figura 1. Propuesta de reforma.

\section{METODOLOGÍA}

En la presente investigación la métodología empleada, se basó según el enfoque cualitativo utilizando la recolección y análisis en una tipología documental bibliográfica, sustentada desde la perspectiva de género descriptiva en función a la determinación de las causas sobre el problema. Los métodos aplicados fueron Inductivo-deductivo, entendiéndose como el razonamiento que, partiendo del análisis del jurídico ecuatoriano, lo cual permitio elevar a conocimientos generales. Histórico Lógico, que está vinculado al conocimiento de las distintas etapas y sucesión cronológica la violencia ecónomica y patrimonial. Analítico - sintético separa el objeto del estudio de (violencia contra la mujer y la familia): el análisis, encargado de 
manejar los juicios, y por otro lado la síntesis que considera los objetos como un todo. La aplicación de todos estos métodos permitió analizar teorías, leyes, jurisprudencias, bibliografías, como parte de la población de estudio, generándose mediante el análisis documental jurídico, la construcción de aportes reflexivos teóricos en el campo del derecho constitucional (Lazo, Erazo y Narváez, 2019).

\section{APORTES GENERADOS}

En virtud de la presente investigación, se puede determinar lo siguiente:

En el Ecuador, no existen suficientes campañas de concientización para la erradicación de la violencia de género, por lo cual los funcionarios públicos y judiciales son quienes deben asegurar y garantizar un trato justo y una atención prioritaria con procesos adecuados y libres de pensamientos misóginos, asumiendo la responsabilidad de brindarles protección para el efectivo goce de sus derechos, fomentado una sociedad más equitativa.

La sociedad actual no ha tomado conciencia con respecto a los derechos de las mujeres debiendo activarse la justica en pro de ellas, con el fin de establecer un procedimiento ágil, oportuno y sin dilaciones evitando así impunidad o un retardo injustificado al momento de comparecer a las entidades estatales, logrando obtener una reparación integral del daño causado con lineamientos a la restitución de los derechos establecidos en nuestro ordenamiento jurídico.

Como política pública de Estado la capacitación en roles de género es de suma importancia para eliminar los estereotipos implantados tales como el machismo y el sistema patrialcal en el cual la mujer a sido víctima, y así lograr la igualdad, equiparación de derechos tanto materiales como inmateriales, la sociedad en valores de género y alejarse de los extremos, buscar el empoderamiento de la mujer, buscando la igualdad cambiando paradigmas sociales y culturales de la ciudadanía.

La violencia económica y patrimonial no solamente es mantener en la casa a la mujer y mantener a la mujer, es la violencia ejercida a sus bienes muebles e inmuebles de los 
recursos económicos en un contexto familiar y social, implementando las vías legales eficientes y eficaces a la justicia que no menoscaben 0 anulen el ejercicio de ese derecho, evitando la revictimización y afianzando la normativa internacional en la no discriminación, garantizando la tutela judicial efectiva.

Una actuación en pro del desarrollo de los derechos humanos fundamentales, es la aplicación de una manera mas explícita y inmediata la figura contravencional para juzgar y sancionar la violencia económica y patrimonial, requiriendo contar con normativa ampliada, eficaz, que desarrolle mejor el tipo de violencia señalada, buscando la erradicación de la problemática sobre las diferentes agresiones que sufre las mujeres, esta responsabilidad debería ser cumplida a cabalidad por los funcionarios que administran justica de manera directa en garantía de los derechos constitucionales.

\section{REFERENCIAS CONSULTADAS}

1. Aldana Zavala, J., \& Valles González, E. (2018). La mujer en Derecho a una Vida libre de violencia desde la Organización Comunitaria. IUSTITIA SOCIALIS, 3(5), 110-127. doi:http://dx.doi.org/10.35381/racji.v3i5.171

2. Almeras, D., \& Calderón, C. (2012). Si no se cuenta no cuenta. Chile: CEPAL.

3. Asamblea Nacional Constituyente. (20 de octubre de 2008). Constitución de la República del Ecuador. Derechos de las personas y grupos de atención prioritaria. Montecristi, Manabí, Ecuador: Registro Oficial 449 de octubre 2008.

4. Asamblea Nacional Constituyente. (20 de octubre de 2008). Constitución de la República del Ecuador. Derechos de protección. Montecristi, Manabí, Ecuador: Registro Oficial 449 de octubre 2008.

5. Asamblea Nacional del Ecuador. (05 de febrero de 2018). Codigo Organico Integral Penal. Contravención de violencia contra la mujer o miembros del núcleo familiar. Quito, Pichincha, Ecuador: Registro Oficial Suplemento 180 de 10-feb.2014.

6. Asamblea Nacional del Ecuador. (05 de Febrero de 2018). Ley Organica Integral para prevenir y erradicar la violencia contra las mujeres. Tipos de Violencia. Quito, Pichincha, Ecuador: Registro Oficial Suplemento 175 de 05-feb.-2018. 
7. Benhabib, S. (1992). Una revisión del debate sobre las mujeres y la teoria moral. Isegoría, 38.

8. Butler, J. (2018). El Género en disputa. Argentina: PAIDÓS.

9. Congreso Nacional. (29 de noviembre de 1995). La Ley Contra la Violencia a la Mujer y la Familia. Titulo Preliminar. Quito, Pichincha, Ecuador: Registro Oficial № 839 del 11 de diciembre de 1995.

10. Congreso Nacional. (29 de noviembre de 1995). La Ley Contra la Violencia a la Mujer y la Familia. Formas de Violencia Intrafamilar. Quito, Pichincha, Ecuador: Registro Oficial № 839 del 11 de diciembre de 1995.

11. Deere, C. D., Contreras, J., \& Twyman, J. (2014). Patrimonial Violence a Study of Womens Property Rights in Ecuador. Latin American Perspectives, 41(1), 143.

12. Díaz, N. Q. (2019). Economías feministas, social y solidaria. Respuesta heterodoxas a la crisis de reproducción en América Latina. Ciencias Sociales, 79.

13. Facio, A., \& Fries, L. (2005). Feminismo, género y patriarcado. Revista sobre enseñanza del Derecho de Buenos Aires, 259.

14. Grijalva Jimenez, A. (2012). Constitucionalismo en el Ecuador. Quito: V\&M Graficas.

15. Guzmán, V. A. (2010). El derecho a la tutela judicial efectiva: una aproximación a su aplicación por los tribunales ecuatorianos. Revista de Derecho, N 14 UASB.

16. Lazo Torres, N. S., Erazo Álvarez, J. C., \& Narváez Zurita, C. I. (2019). El Balanced Scorecard como herramienta de control interno en el sector Manufacturero. Koinonía, 131.

17. López, O. C. (2017). La violencia económica y/o patrimonial contra las mujere en el ámbito familiar. Instituto de la Familia Facultad de Derecho, 42.

18. Munster Infante, B. (2017). Género y Sociedad Encrucijadas Teóricas y Alternativas para el Cambio. La Habana: Universitaria Félix Valera.

19. Naciones Unidas. (2002). Violencia contra la mujer en relación de pareja: América Latina y el Caribe Una propuesta para medir su magnitud y evolución. Uso de Indicadores de Género para la Formulación de Políticas Públicas, 14.

20. Observatorio de Igualdad de Género de América Latina y el Caribe. (2019). Autonomía física. Obtenido de Leyes de violencia: https://oig.cepal.org/es 
21. Prieto, M. (2005). Mujeres ecuatorianas entre las crisis y las oportunidades 19902004. Quito: Consejo Nacional de las Mujeres CONAMU.

22. Proveyer Cervantes, C., \& Romero Almodovar, M. (2017). Género y Sociedad Encrucijadas Téoricas y Alternativas para el Cambio. La Habana: Universitaria Félix Valera.

23. Sendón de León, V. (2003). Mujeres en la era global. Barcelona: Icaria más madera.

24. Sistema de Información Científica. (2015). Las mujeres separadas de cara a la violencia de sus exparejas. Rumbo a su caracterización. ed de Revistas Científicas de América Latina, el Caribe, España y Portugal Proyecto académico sin fines de lucro, desarrollado bajo la iniciativa de acceso abierto, 88.

(C2020 por los autores. Este artículo es de acceso abierto y distribuido según los términos y condiciones de la licencia Creative Commons Atribución-NoComercial-Compartirlgual 4.0 Internacional (CC BY-NC-SA 4.0) (https://creativecommons.org/licenses/by-nc-sa/4.0/). 